\title{
Recent advances in ultrasound-based transdermal drug delivery
}

This article was published in the following Dove Press journal:

International Journal of Nanomedicine

\section{Brenden Cheong-Qi Seah Boon Mian Teo}

School of Chemistry, Monash University, Clayton, VIC 3800 , Australia
Correspondence: Boon Mian Teo School of Chemistry, Monash University, 19 Rainforest Walk, Clayton, VIC 3800 , Australia

Tel +6I 399051097

Email boonmian.teo@monash.edu

\begin{abstract}
The transdermal transport of pharmaceuticals possesses various advantageous properties over conventional drug administration techniques such as oral delivery and hypodermic injections. However, the stratum corneum persists as the main barrier, which impedes percutaneous transport. The ultrasound-based transdermal delivery of therapeutics is one of the techniques that are being investigated to overcome this obstacle. This review outlines the background information pertaining to sonophoresis and then discusses the individual sections of sonophoretic research. These areas include the sonophoretic application of various drugs, dualfrequency sonophoresis, synergistic combinations of transdermal drug delivery techniques, and the use of nanosized carriers in ultrasound-based transdermal delivery. The various challenges associated with sonophoretic drug delivery and trends of future research are also highlighted.

Keywords: sonophoresis, acoustic cavitation, microneedle, ultrasound, nanoparticles
\end{abstract}

\section{Introduction}

Transdermal delivery of drugs has made a substantial contribution in the field of biomedical applications, but it has not fully achieved its potential as an alternative to the oral and intravenous dominated techniques of drug administration. This contemporary method would be an attractive and beneficial addition to the possible routes of drug administration. Oral medication is commonly used for its convenience, but absorptive inefficiencies impede its pharmaceutical capacity. ${ }^{1}$ Contrarily, hypodermic injections allow an accurate dose of medication to be delivered directly to the systemic circulation, but this method of drug delivery also carries several disadvantages. Up to $30 \%$ of adults suffer from needle phobias due to the associated pain. ${ }^{2}$ In addition, the risk of unintentional needle injury to health professionals and patients substantiates the undesirable transmission of infective diseases. ${ }^{2}$ Transdermal drug delivery offers several advantages to address issues associated with oral delivery and hypodermic injections through the application of specialized medicated adhesive patches onto the skin. ${ }^{3}$ However, there are several limitations associated with transdermal drug delivery systems. The efficacy and implementation of transdermal drug delivery is principally limited by the physical barrier of the skin. The human epidermis has numerous stratum layers that are maintained by keratinocytes. Proliferation of these cells causes the mature cells to migrate toward the outer layer of the epidermis, the stratum corneum (SC). This outer barrier is composed of a 15-20 $\mu \mathrm{m}$ thick layer of keratin-filled dead corneocytes that have a "brick and mortar" structure (Figure 1). ${ }^{2}$ Potent permeation through this natural fortification is being perpetually investigated through various novel techniques. Some studies have explored third-generation methods including chemical enhancers, microneedles, thermal ablation, and ultrasound. ${ }^{4}$ This review introduces the reader 


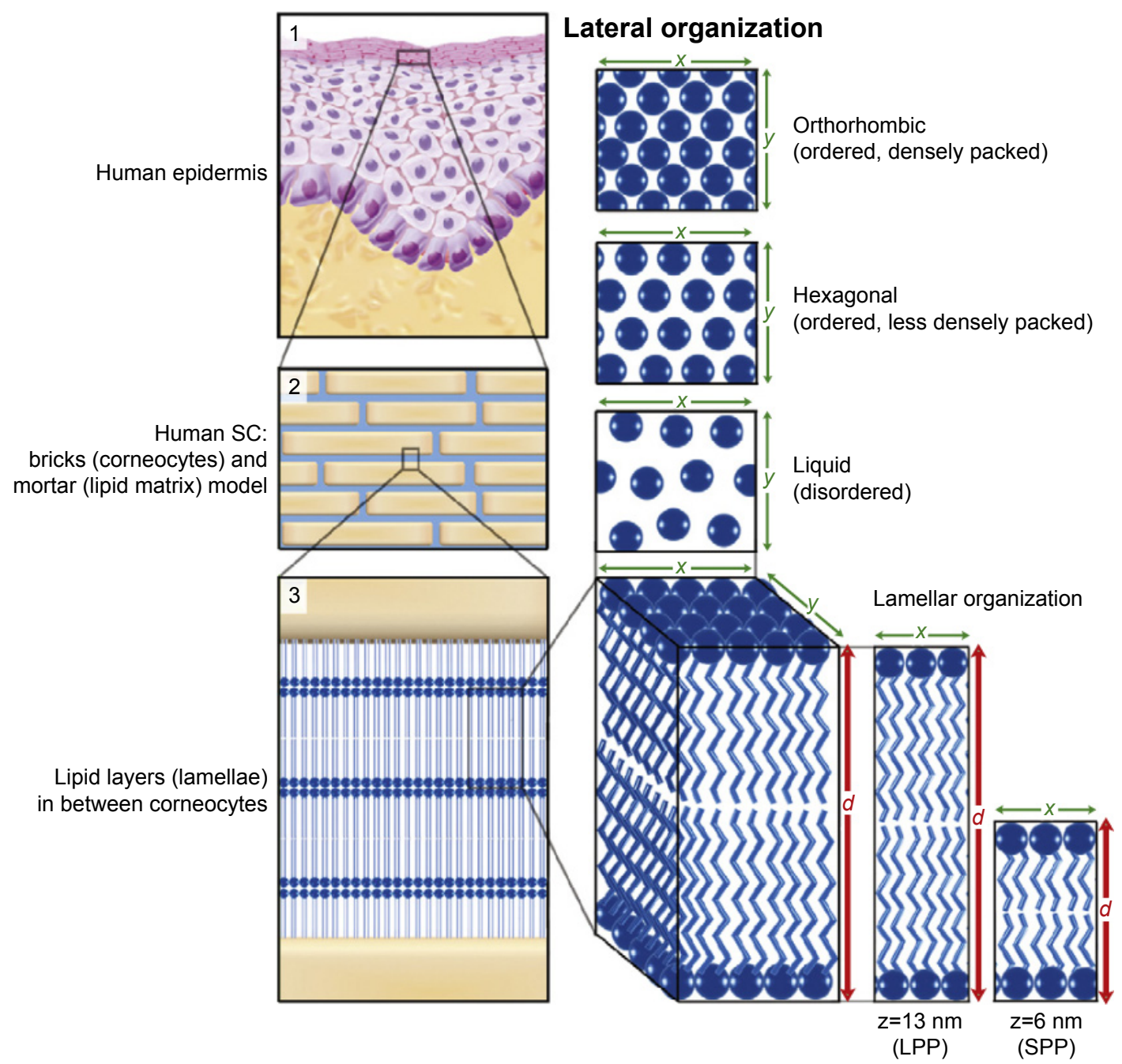

Figure I Lipid organization in human SC.

Notes: (I) The outermost layer of the epidermis, the SC, consists of dead cells (corneocytes) embedded in a lipid matrix, also referred to as the brick (corneocytes) and mortar (lipids) structure (2). The intercellular lipids are arranged in layers (lamellae; 3$)$, with either a long or short repeat distance (d) with LPP $(\mathrm{z}=13 \mathrm{~nm})$ or SPP $(\mathrm{z}=6 \mathrm{~nm})$, respectively. The lateral organization is the plane perpendicular to the direction of the lamellar organization. There are three possible arrangements of the lipids: a very dense, ordered orthorhombic organization; a less dense, ordered hexagonal organization; or a disordered liquid organization. Reproduced from Biochimica et Biophysica Acta (BBA)-Molecular and Cell Biology of Lipids, I84I(3), Van Smeden J, Janssens M, Gooris GS, Bouwstra JA. The important role of stratum corneum lipids for the cutaneous barrier function, 295-3/3. Copyright (2014), with permission from Elsevier. ${ }^{66}$

Abbreviations: LPP, long periodicity phase; SC, stratum corneum; SPP, short periodicity phase.

to ultrasound-based transdermal drug delivery. For readers interested in alternative third-generation transdermal drug delivery methods, Alkilani et $\mathrm{al}^{1}$ and Schoellhammer et $\mathrm{al}^{2}$ have conducted illuminating reviews on various additional investigated techniques. The reader will be first introduced to the basics of ultrasound for understanding the theoretical application and improvement of this drug administration technique. The review then proceeds to evaluate recent improvements and limitations and future challenges within this field of research.

\section{Ultrasound}

The fundamental mechanism responsible for the functional capacity of ultrasound is the manipulation of mechanical energy. This energy is transported as acoustic waves that surpass the audible human range. It is a commonly utilized modern medical imaging modality on account of its convenience, economical cost, and nonionizing and relatively noninvasive attributes. Ultrasound also holds a unique mechanical influence on gas bubbles, which allows the manipulation and utilization of cavitation effects within the field of ultrasound-based transdermal drug delivery.

\section{Physics of ultrasound}

The manipulation of mechanical energy occurs at the piezoelectric crystals within a transducer, where an alternating current is converted into mechanical vibrations ${ }^{5}$ and transmitted into the patient. As these vibrations are generated at 
the ultrasound probe, there is essentially a transfer of energy occurring along a directed path. The mechanical energy propagates into the body longitudinally in oscillations of high (compression) pressure and low (rarefaction) pressure. The produced ultrasound wave has an explicit frequency, which is the number of compression peaks each second. ${ }^{6}$ As depicted in Equation 1, this frequency $(f)$ correlates with a certain wavelength $(\lambda)$ by factoring the velocity (c), where the average speed of sound in human tissue is $\sim 1,540 \mathrm{~m} / \mathrm{s}^{7}$

$$
\left(\lambda=\frac{c}{f}\right) .
$$

The wavelength of a sound wave is defined as the distance a sound wave travels in one cycle ${ }^{7}$ from one point of peak compression to another. Higher frequencies waves have smaller wavelengths and yield superior spatial resolution of $\sim 0.5 \mathrm{~mm}$, but at the sacrifice of reduced penetrability. ${ }^{8}$

\section{Bioeffects of ultrasound}

Attenuation is another fundamental principle involved in the physics of ultrasound. As the generated mechanical energy passes through the tissue, some of the energy will be lost along its path due to four main causes: reflection, diffraction, refraction, and absorption. ${ }^{9}$ The absorption of ultrasound waves into a material with a high acoustic impedance will readily convert mechanical energy into heat through the friction between tissue particles that are oscillating. Other factors influencing temperature include convection, conduction, the ultrasound exposure parameters, and exposure duration. ${ }^{10}$ This production of heat is the biological effect of ultrasound, which is important to be aware of. To measure this component of probable bioeffects, the thermal index is used to provide an indication for the potential increase in temperature. ${ }^{11}$

Mechanical effects of ultrasound involve interactions of mechanical energy with gas bubbles. ${ }^{12}$ As ultrasound waves pass through the tissue, gas bubbles can undergo expansion and contraction due to rectified diffusion and bubble coalescence. ${ }^{13}$ This is defined as the process of acoustic cavitation and can be categorized into stable cavitation or inertial cavitation. ${ }^{14}$ Stable cavitation occurs when a lower ultrasound intensity causes a conservative oscillation in bubble volume. Contrarily, a higher ultrasound intensity provokes the oscillating bubbles to reach their maximum potential size and eventually collapse. ${ }^{15}$ Resulting physical effects attributed to inertial cavitation are shock waves or microjets. Wolloch and $\operatorname{Kost}^{16}$ surmised that particles with larger diameter of $250 \mu \mathrm{m}$ induced microjetting, while smaller particles with diameter of $10 \mu \mathrm{m}$ generated more shock waves. The symmetrical collapse of a bubble produces a shock wave that evenly distributes the release of energy into the surrounding tissue (Figure 2). ${ }^{17}$ Conversely, immense nonspherical oscillations lead to an unstable nucleation with the influential phenomenon of microjetting. The asymmetrical bubble collapse near a large extrinsic surface causes the bubble to "turn in" on itself ${ }^{13}$ and consequently generate a high-speed liquid microjet with velocities up to $100 \mathrm{~m} / \mathrm{s},{ }^{18}$ where the distribution of energy becomes concentrated to a particular point (Figure 2). To measure this preeminent bioeffect, the mechanical index was introduced by Holland and Apfel $^{19}$ in 1989 to evaluate the capability to induce cavitation and consequently cause reversible damage to tissue. ${ }^{20}$ As seen in Equation 2, the mechanical index (MI) is determined by the peak rarefactional acoustic pressure $(P-)$ and the operating frequency $(f) .^{21}$

$$
\left(\mathrm{MI}=\frac{P-}{\sqrt{f}}\right) .
$$

\section{Sonophoretic effects on the skin}

The use of ultrasound for delivery of active ingredients across the skin barrier was first reported in 1954, where hydrocortisone was used to treat digital polyarthritis. ${ }^{22}$ Among the available literature, there have been various theorized mechanisms on how acoustic cavitation affects the skin. The identified mechanical effects of acoustic cavitation have been attributed to play a significant role in increasing skin permeability, but the exact method of how it works is still not understood. ${ }^{23}$ Some have hypothesized that the shock waves structurally modify lipids of the SC to produce diffusion channels, while the high-pressure microjets physically disrupt the lipid bilayers (Figure 1) of the SC. ${ }^{24}$ Alternative theories suggest that the process of sonophoresis (SP) is attributed to microstreaming flow in close proximity to the skin, which provokes shear stresses to stretch the SC and to produce channels for transdermal delivery. ${ }^{25,26}$ Thermal effects of ultrasound have also been speculated to positively influence sonophoretic skin interaction through increases in the drug diffusion coefficient and skin permeability coefficient. ${ }^{18}$ However, the practical application of SP may provide insufficient intensity to significantly impact the molecular kinetic energy of the influenced drugs and tissue. In addition, heating damage could occur to thermally unstable transdermal drugs. ${ }^{18}$ 


\section{A} Isotropic bubble collapse and shock wave propagation
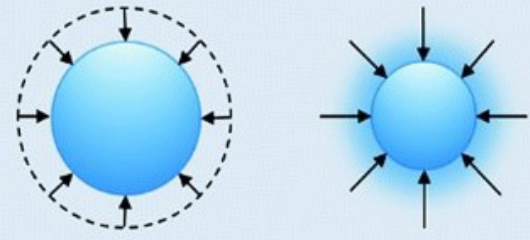

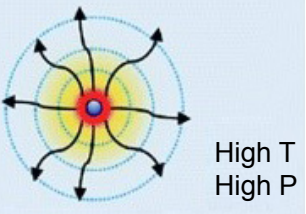

Bubble collapse and shock wave propagation

B Anisotropic cavitation of microjet at interfaces

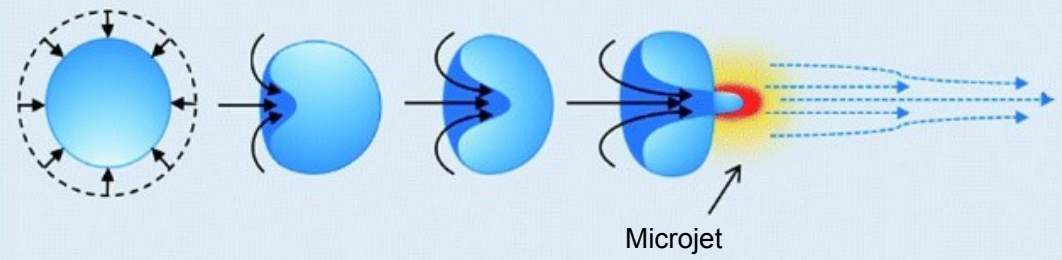

Figure 2 Diagram of $(\mathbf{A})$ a shock wave produced by symmetrical bubble collapse and $(\mathbf{B})$ the asymmetrical bubble collapse generating a microjet. Notes: $\mathrm{T}$ and $\mathrm{P}$ represent temperature and pressure, respectively. Reproduced with permission from Royal Society of Chemistry. Chan Kim Y, Min $\mathrm{H}$, Yu J, et al. Forced infiltration of silica beads into densely-packed glass fibre beds for thin composite laminates. RSC Adv. 2016;6(94):91341-91348. ${ }^{17}$

\section{Single-frequency SP of drugs}

The sonophoretic delivery of drugs relies on the generation of microjets from acoustic cavitation to increase skin permeability and allow the transportation of molecules through small channels. Considerable prior and current researches into sonophoretic drug delivery have been concentrated on better understanding the underlying mechanisms, optimization of transport, and successfully delivering a broader range of remedial drugs transdermally. ${ }^{24}$ Several studies in the field of ultrasound-mediated transdermal delivery have been conducted using a range of therapeutic compounds, and of these studies, many have examined the potential for transdermal delivery of insulin due to the high prevalence, cost, and inconvenience of diabetic treatments. ${ }^{27}$ One of the recent insulin-related studies conducted by Feiszthuber et $\mathrm{al}^{28}$ examined the potential for transdermal transport of insulin into skin agar models and porcine skin in vitro using SP. Their results illustrated that in both skin models, there was a significant increase in the percutaneous delivery of insulin, but only the agar model displayed an increased penetration depth. Another study by Jabbari et $\mathrm{al}^{29}$ attempted to develop and test a portable, low-cost ultrasound transducer for the sonophoretic delivery of insulin. The developed ceramic transducer was used to transmit a pulsed frequency of $40 \mathrm{kHz}$ over 60 minutes to transdermally deliver insulin to rats in vivo. Their study also involved applying various other drug delivery techniques, such as subcutaneous injection and topical treatment, to correlate the potency of transdermal insulin transport. To determine the effectiveness of each method, blood glucose levels were recorded before treat-

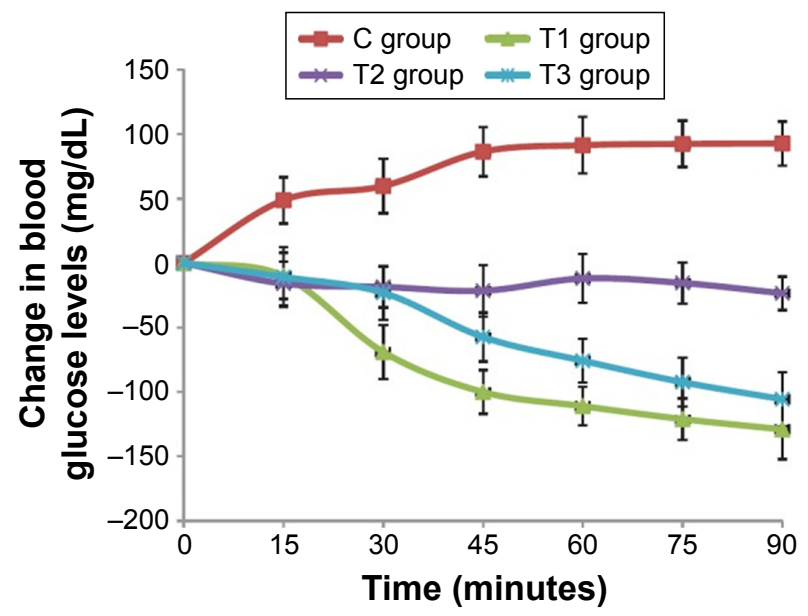

Figure 3 Change in blood glucose levels $(\mathrm{mg} / \mathrm{dL})$ of rats in vivo with respect to time (minutes).

Note: Reproduced from Jabbari N, Asghari MH, Ahmadian H, Mikaili P. Developing a Commercial Air Ultrasonic Ceramic Transducer to Transdermal Insulin Delivery. J Med Signals Sens. 2015;5(2):117-122. Creative Commons license and disclaimer available from: http://creativecommons.org/licenses/by/4.0/legalcode. ${ }^{29}$ 
A

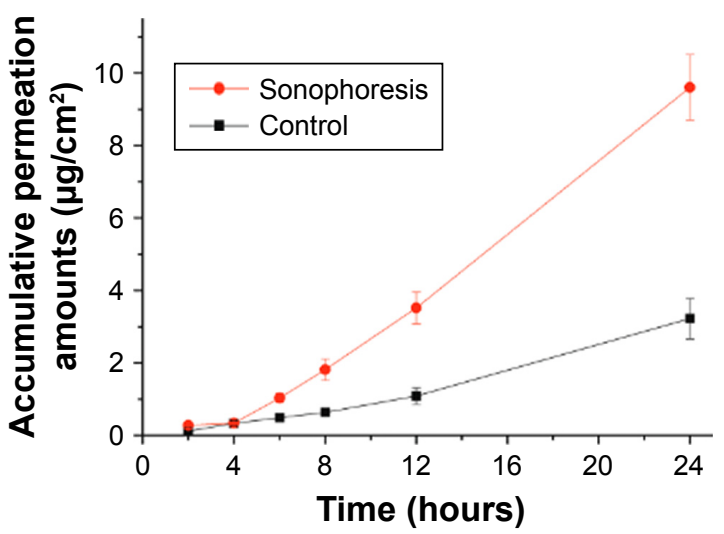

B

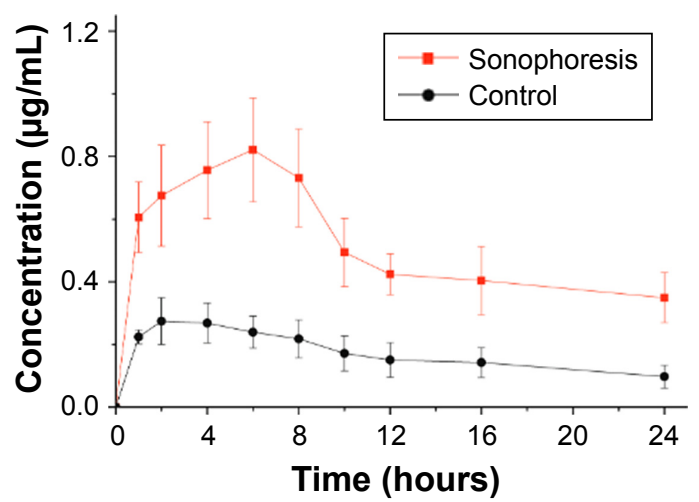

Figure 4 (A) Transdermal profiles of rivastigmine permeation into in vitro pig skin. (B) Plasma concentration curves of rivastigmine in pigs in vivo with respect to time. Note: Reproduced with permission from Avoxa-Mediengruppe Deutscher Apotheker GmbH. Yu ZW, Liang Y, Liang WQ. Low-frequency sonophoresis enhances rivastigmine permeation in vitro and in vivo. Pharmazie. 20I5;70(6):379-380.30

ment and every 15 minutes after treatment. As shown in Figure 3, they found that while the injection group (T1) and the sonophoretic group (T3) exhibited no significant difference, there was a significant difference between the sonophoretic group and the topical group (T2). In addition, the authors perceived no consequential change or damage to the insonified skin.

While the transdermal delivery of insulin remains a major and active area of research, additional studies have examined the prospects of transporting other drugs transdermally. A study by $\mathrm{Yu}$ et $\mathrm{al}^{30}$ compared commercially available rivastigmine transdermal patches with the sonophoretic delivery of rivastigmine using an ultrasound frequency of $20 \mathrm{kHz}$. The permeability of each method was tested on porcine skin in vivo and in vitro. By using high-performance liquid chromatography, they established that the in vitro sonophoretic group displayed significant enhancement of rivastigmine permeated by a factor of 3.1 , when compared to the in vitro control samples (Figure 4A). Blood samples collected from pigs in vivo generated similar results, indicating $298 \%$ enhancement in bioavailability of rivastigmine plasma concentration in the sonophoretic group (Figure 4B).

Pereira et al ${ }^{31}$ delivered doxorubicin transdermally into porcine skin in vitro using ultrasound, while attempting to enhance skin permeability through the application of various coupling mediums. These solutions included poloxamer, hydroxyethyl cellulose, chitosan, and carbopol hydrogels, in addition to sodium lauryl sulfate solution. An ultrasound frequency of $20 \mathrm{kHz}$ was utilized, and the permeated doxorubicin was measured 26 hours after sonication. Within their findings, the only statistically significant result obtained was from the carbopol sample, which displayed significantly more doxorubicin transported into the SC (Figure 5). The authors also observed that the doxorubicin transport ratio of viable epidermis to the $\mathrm{SC}$ was 0.7 in the carbopol sample, while the other hydrogels held ratios between 1.4 and 2 .

Another potential drug examined by Aldwaikat and Alarjah was diclofenac sodium. ${ }^{32}$ Their study used several combinations of ultrasound parameters, such as frequencies of $1 \mathrm{MHz}$ or $20 \mathrm{kHz}$, varying amplitudes, assorted sonication times, and pulsed vs continuous waves. Diclofenac sodium was transdermally delivered into numerous "EpiDerm" skin cultures, and the flux of each sample was documented and compared in Figure 6A and B. They found that using a frequency of 20 $\mathrm{kHz}$ in a continuous mode for 5 minutes generated statistically significant flux averages compared to that in the control, particularly for the $20 \%$ amplitude sample, which held a $546 \%$ increase over the control. However, there was no significant

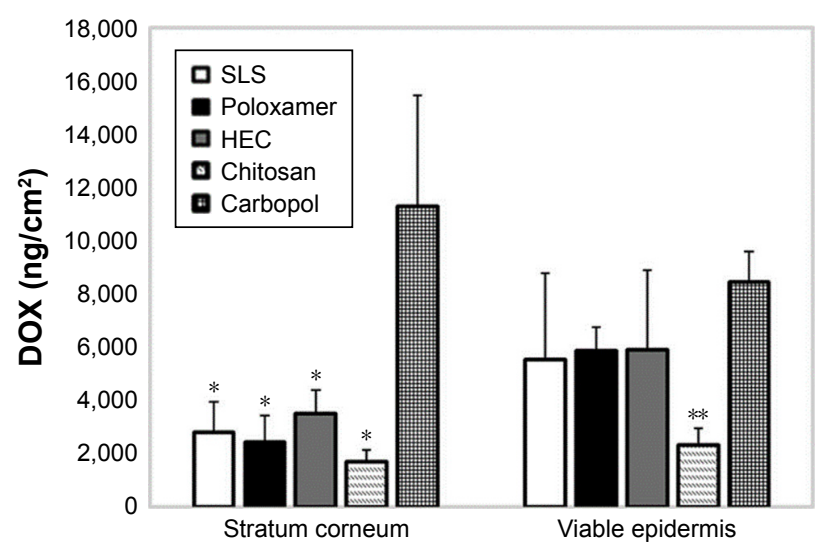

Figure 5 Concentrations of DOX delivered to the skin after low-frequency ultrasound treatment using hydrogels as the coupling medium.

Notes: The results were analyzed according to ANOVA followed by post hoc analysis using Tukey's test with $p<0.05$ as the minimum level of significance. SC: ${ }^{*} p<0.05$ vs carbopol; viable epidermis: ${ }^{*} p<0.05$ vs carbopol. Reproduced from Pereira TA, Ramos DN, Lopez RF. Hydrogel increases localized transport regions and skin permeability during low frequency ultrasound treatment. Sci Rep. 20 I7;7:44236. Creative Commons license and disclaimer available from: http://creativecommons. org/licenses/by/4.0/legalcode. ${ }^{31}$

Abbreviations: DOX, doxorubicin; HEC, hydroxyethyl cellulose; SC, stratum corneum; SLS, sodium lauryl sulfate. 
A

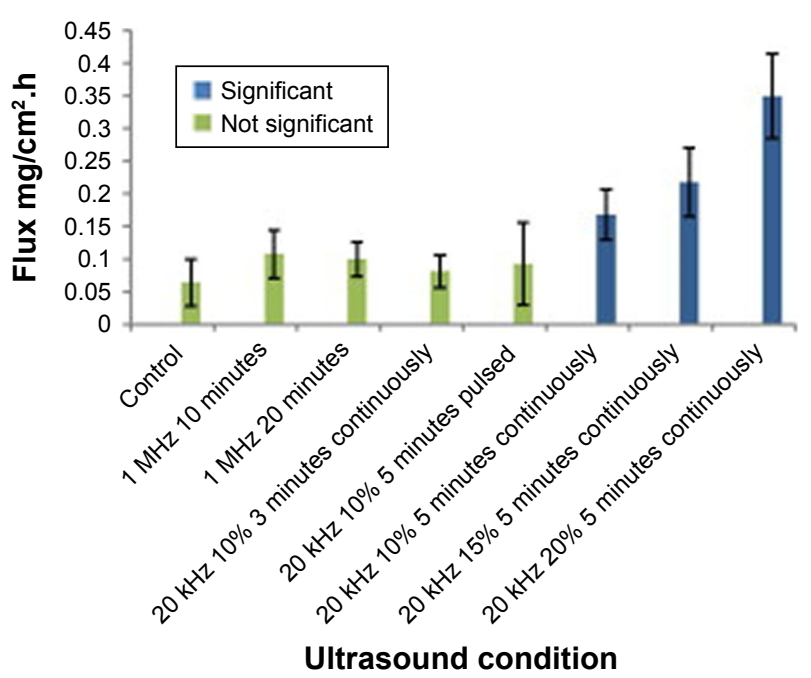

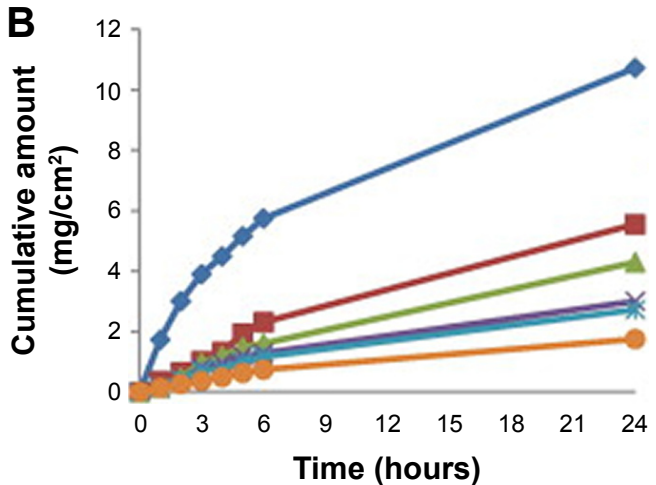

Time (hours)

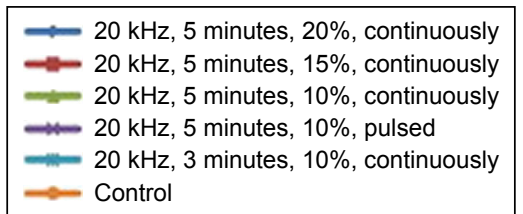

C
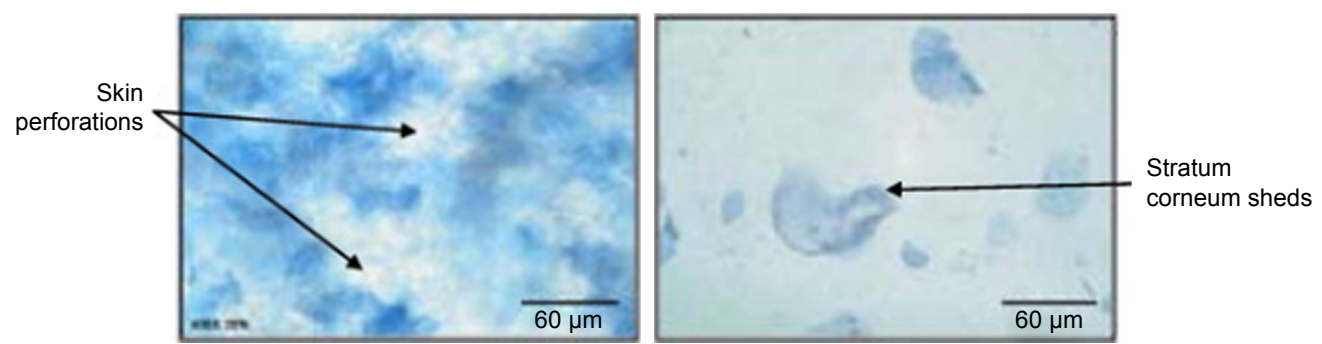

Figure 6 (A) Diclofenac sodium flux into skin cultures vs ultrasound parameter configuration. (B) Concentration of diclofenac sodium permeated vs time at various ultrasound amplitudes. (C) Histological image of skin culture insonified with $20 \mathrm{kHz}$ continuously with a duty factor of $20 \%$ for 5 minutes. ${ }^{32}$

Note: Reproduced from Ultrason Sonochem, 22, Aldwaikat M, Alarjah M. Investigating the sonophoresis effect on the permeation of diclofenac sodium using 3D skin equivalent, 580-587. Copyright (2015), with permission from Elsevier.

difference between the $15 \%$ and $10 \%$ amplitude samples. After sonication of the skin cultures, the samples were also histologically examined. They found that increasing amplitude of continuous mode ultrasound induced shedding of the SC (Figure 6C), while pulsed mode did not display shedding. An additional skin culture was insonified with an amplitude of $50 \%$ at $20 \mathrm{kHz}$ for 5 minutes, and the SC damage of this sample was so exorbitant that permeation of diclofenac sodium could not be measured. The collective results of these studies demonstrate that transdermal drug delivery is an astonishingly effective and noninvasive method to deliver drugs when compared with the current traditional options. However, it is also imperative to consider the potential thermal and mechanical bioeffects of SP. Further augmentation of this transdermal delivery technique through increasing the SP intensity or duration may result in detrimental adverse reactions.

\section{Dual-frequency SP}

Typically, to increase percutaneous transport in single-frequency SP, it would require an increase in ultrasound treatment duration or intensity, but recent studies have found that dualfrequency SP is able to enhance transdermal transport, while minimizing thermal effects. The predominant observed configuration for dual-frequency SP positioned the low-frequency transducer perpendicular to the surface, with the high-frequency transducer arranged at a $90^{\circ}$ angle to the low-frequency transducer. A study by Schoellhammer et al ${ }^{33}$ investigated the potential for dual-frequency ultrasound to improve skin permeability through cavitational activity. Their investigation consisted of two parts and utilized low ultrasound frequencies of 20, 40, and $60 \mathrm{kHz}$ in combination with high frequencies of 1 and $3 \mathrm{MHz}$. For the first component of the study, aluminum foil was prepared and insonified with single- and dual-frequency beams to measure the radius and quantity of microjets generated through acoustic cavitation. As shown in Figure 7A, they found that the dual-frequency ultrasound for low frequencies of 20 and $40 \mathrm{kHz}$ was effective in increasing the total pitted area from acoustic cavitation. They also determined that while $1 \mathrm{MHz}$ dual-frequency ultrasound produced a greater pitted area than $3 \mathrm{MHz}$ dual-frequency ultrasound, the difference was not statistically significant. The second part of their study involved the sonophoretic delivery of glucose and insulin into in vitro porcine skin with single- and dual-frequency ultrasound to validate that the previously ascertained results were applicable 
A

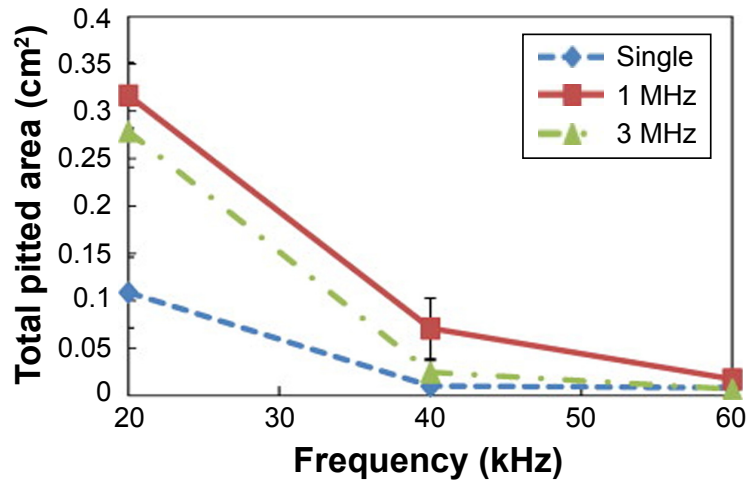

B

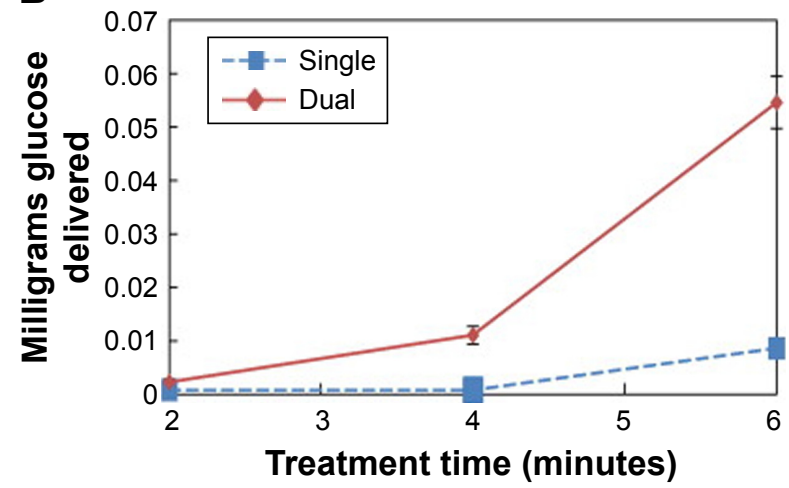

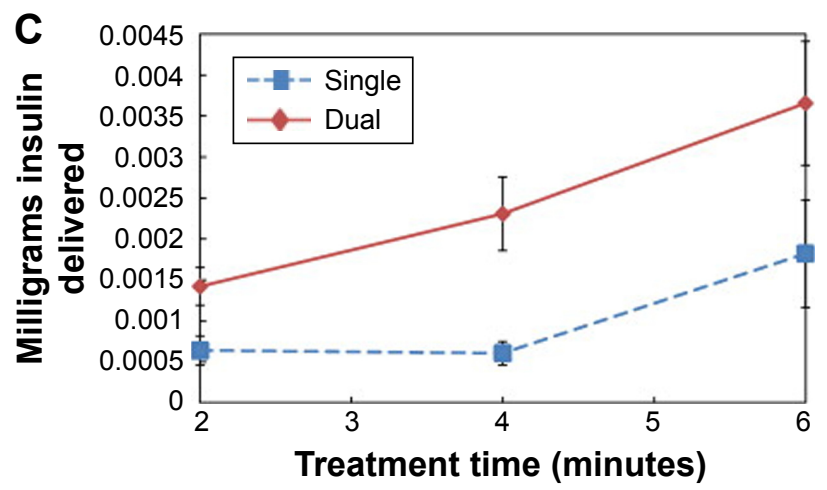

Figure 7 Blue line represents single $20 \mathrm{kHz}$ frequency, while red represents $20 \mathrm{kHz}+\mathrm{I} \mathrm{MHz}$ and green represents $20 \mathrm{kHz}+3 \mathrm{MHz}$.

Notes: (A) Total pitted area of aluminum foil with respect to frequency for configurations of single and dual frequencies. (B) Volume of glucose delivered into porcine skin in vitro vs ultrasound pretreatment time. (C) Volume of insulin delivered into porcine skin in vitro vs ultrasound pretreatment time. Reproduced from J Control Releas, I63(2), Schoellhammer CM, Polat BE, Mendenhall J, et al. Rapid skin permeabilization by the simultaneous application of dual-frequency, high-intensity ultrasound, I54-I60. Copyright (2012), with permission from Elsevier. ${ }^{33}$

to transdermal transport. As the frequencies of $20 \mathrm{kHz}$ and 1 $\mathrm{MHz}$ were observed to display the greatest cavitational activity, they were selected for the sonification of in vitro skin. The collected results validated their hypothesis that dual-frequency SP enhanced the percutaneous transport of glucose (Figure 7B) and insulin (Figure 7C) into the skin.

Schoellhammer et $\mathrm{al}^{34}$ then conducted a follow-up study to establish the relative safety in vitro and verify the capability of dual-frequency ultrasound in vivo. From the previous dual-frequency study, it was established that a higher frequency nucleates the population of smaller bubbles to synergistically couple with the lower frequency. ${ }^{35} \mathrm{By}$ simultaneously using frequencies of $20 \mathrm{kHz}$ and $1 \mathrm{MHz}$ in vivo, they verified that there was an increased skin permeability at a faster rate when compared to using $20 \mathrm{kHz}$ alone (Figure 8A). This was attributed to a possible enhancement of cavitational activity, which consequently produced larger pores for more efficient transport. Furthermore, their in vitro study determined that there was no observable histological difference in epidermal disruption between using dual frequency and low frequency (Figure 8B).

A similar study that utilized frequencies of 20 and $800 \mathrm{kHz}$ investigated the permeability of sinomenine hydrochloride into porcine skin in vivo. ${ }^{35}$ They obtained similar results and concluded that dual-frequency ultrasound was able to significantly increase skin permeability (Figure 9A), while reducing thermal side effects. In addition, they highlighted that both pulsed and continuous modes of ultrasound produced equally effective results for either single or dual frequency (Figure 9B). As a result, they recommend the employment of pulsed mode ultrasound in SP as a supplementary source of minimizing potential thermal damage.

While dual-frequency ultrasound displays promising influences for sonophoretic transdermal drug delivery, there remain substantial challenges that impede its efficacy and implementation. First, the optimization of a dual-frequency approach is largely unknown, ${ }^{36}$ with studies postulating frequency combinations. Second, the interaction of bubbles with dual frequency is not sufficiently understood. To investigate these issues, a group of authors recently conducted various studies that theoretically analyzed bubble dynamics under dual-frequency ultrasound to examine the effects of multiple simultaneous frequency sonification. ${ }^{37}$ They discovered the presence of combination and simultaneous resonances, which are 
A

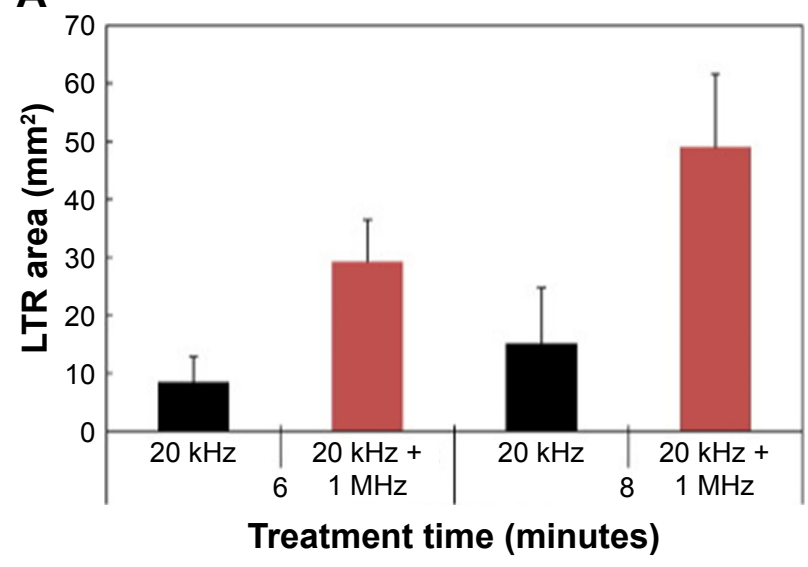

B
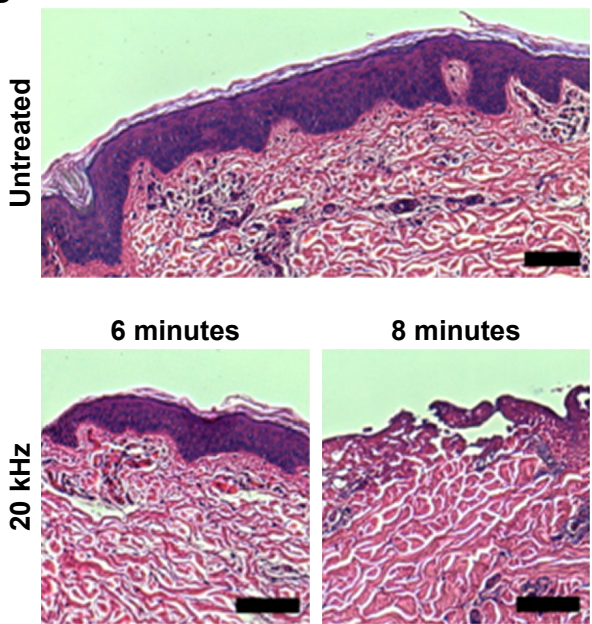

8 minutes
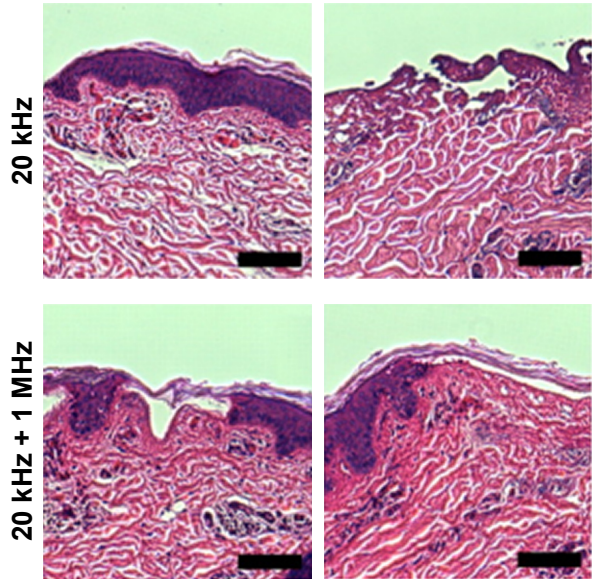

Treatment time (minutes)

Figure 8 (A) Comparison of localized transport region size vs treatment time in vivo for single- and dual-frequency sonophoresis. (B) Histological images of in vitro porcine skin treated with single- and dual-frequency sonophoresis.

Notes: Reproduced from J Control Release, 202, Schoellhammer CM, Srinivasan S, Barman R, et al. Applicability and safety of dual-frequency ultrasonic treatment for the transdermal delivery of drugs, 93-100. Copyright (2015), with permission from Elsevier. ${ }^{34}$ Scale bar in all images is $50 \mu \mathrm{m}$.

Abbreviation: LTR, localized transport regions.

unique to dual-frequency ultrasound, and further examined the applicability of dual-frequency ultrasound to control chaotic bubble oscillations. ${ }^{38}$ The authors emphasized that the regulation of chaotic bubble oscillations allowed consistent manipulability of acoustic cavitation effects and consequently enhanced sonophoretic percutaneous transport. These studies on bubble dynamics under the influence of dual-frequency ultrasound can allow further

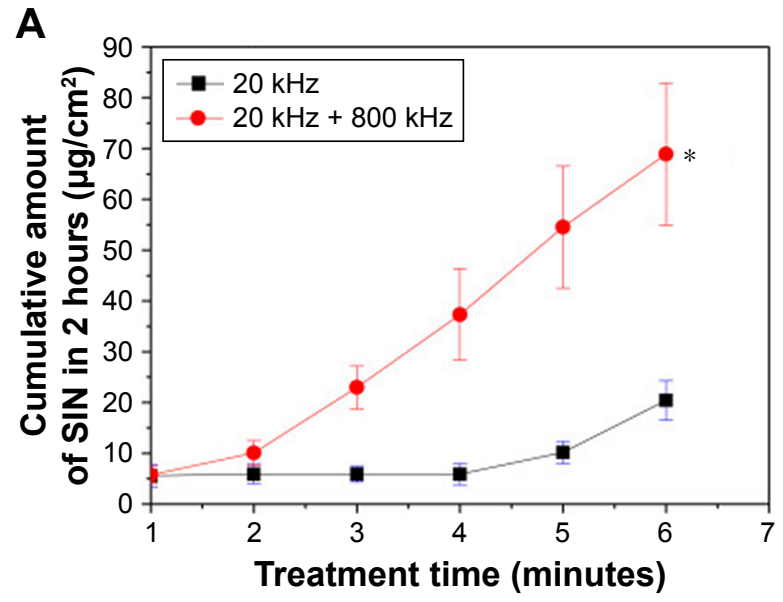

B

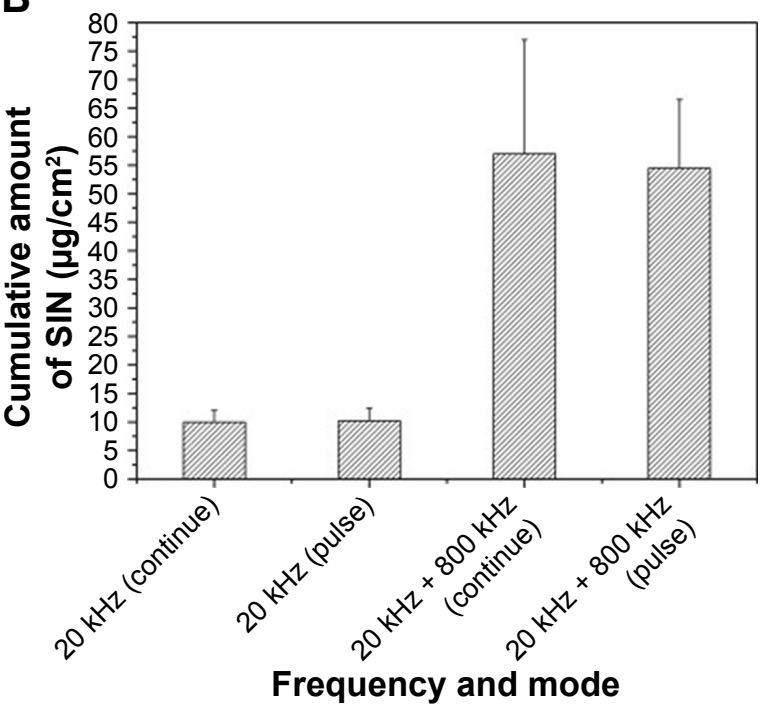

Figure 9 (A) Concentration of SIN hydrochloride permeated into pig skin in vivo in 2 hours vs treatment time for single- vs dual-frequency sonophoresis. (B) Comparison of SIN hydrochloride permeated for pulsed $50 \%$ duty cycle vs continuous ultrasound operation using single or dual frequency.

Notes: Reproduced from J Drug Deliv Sci Technol, 36, Yin L, Qin FH, Zhou Y, Qi X, Enhancing percutaneous permeability of sinomenine hydrochloride using dual-frequency sonophoresis, 62-67. Copyright (2016), with permission from Elsevier..$^{35}$ Error bars represent one standard deviation. ${ }^{*} p<0.05$ versus $20 \mathrm{kHz}$ single frequency group. Abbreviation: SIN, sinomenine. 
research into the enhanced control of acoustic cavitation effects for sonophoretic transdermal drug delivery.

\section{Combining transdermal drug delivery techniques}

Another intriguing area of sonophoretic research involves the collaborative use of multiple transdermal drug delivery techniques to optimize skin permeability and enhance macromolecule transport. ${ }^{24}$ The foremost transdermal drug delivery method is the synergistic application of microneedles with SP. Microneedles are another form of transdermal drug delivery method that has also undergone significant development and research. There are various iterations of microneedles with an assortment of needle sizes and composing materials, such as stainless steel or silicon, ${ }^{39}$ but the fundamental design comprises a microneedle array arranged onto a patch. Currently, there are four main microneedle designs, each of which have specific mechanisms to induce percutaneous delivery. A solid array of microneedles serves the sole purpose of penetrating the skin to increase permeability, allowing for the transdermal transport of topical agents. Degradable microneedles are composed of safe biodegradable polymer needles that slowly release the drug into the skin. Hollow microneedles passively or actively inject a liquified drug through hollow bores that run down the center of the microneedle. Coated microneedles use metallic needles that are coated with the medication..$^{40}$ Electroporation (EP) is another method of transdermal drug delivery that has been investigated for its synergistic effects with SP. EP utilizes high voltage pulses of electricity to establish aqueous pores, allowing for percutaneous transport. The degree of skin permeability precipitated from EP is dependent on particular characteristics of the electric pulses. These characteristics include the duration, amplitude, shape, and number of electric pulses applied, but it has been found that amplitude and pulse length are the predominant influential electrical attributes. Finding an optimal balance between these various parameters to induce maximal skin permeability has been a focal area of EP research. ${ }^{41}$ An additional investigated electrical percutaneous delivery technique is iontophoresis (IP), which uses weaker electric currents than that used in EP. ${ }^{42}$ IP permits transdermal drug delivery though the manipulation of ions. This occurs through processes called electromigration and electro-osmosis. ${ }^{43} \mathrm{By}$ combining these transdermal delivery techniques with SP, percutaneous transport may be complementarily augmented and maximized.

Research by Pamornpathomkul et $\mathrm{al}^{44}$ examined the effects of combining low-frequency SP with microneedles in the in vitro transdermal delivery of fluorescein isothiocyanate-dextrans. By using confocal laser scanning microscopy, they determined that the magnitude of fluorescence was greatest when the two transdermal drug delivery techniques were combined, when compared with using each method individually. A comparable study by Petchsangsai et $a l^{65}$ tested each possible combination in three identified transdermal delivery methods: SP, microneedles, and EP for the percutaneous delivery of flurorescein isothiocyanate-dextrans in vitro. The techniques were tested individually, paired, and then grouped. Their results also indicated that combined transdermal drug delivery techniques worked synergistically to improve skin permeability, with the incorporation of all three techniques generating the largest amount of fluorescein isothiocyanate-dextrans transport of $16.58 \pm 2.35 \mu \mathrm{g} / \mathrm{cm}^{2} / \mathrm{h}$. The permeability in combining various transdermal delivery techniques was found to be nearly 100 times greater than that of SP alone. In addition, this enhanced permeability may also minimize the potential for inducing various bioeffects from SP. In an elegant review by Han and Das, they have introduced the relevant mechanisms of microneedles and ultrasound-based transdermal drug delivery and have also pointed out the weak areas of these two technologies. They concluded that the combination of both microneedles and ultrasound allows higher and controllable transdermal delivery rate. ${ }^{45}$

Conversely, a study by Tokumoto et $\mathrm{a}^{46}$ combined IP with EP or SP to construe their effects on electro-osmosis. To determine the electro-osmotic activity, mannitol was transdermally delivered into mouse skin. The sonophoretic component of this study involved using a frequency of $20 \mathrm{kHz}$ in pulsed mode. While the pretreatment of EP displayed no significant enhancement to mannitol flux, the combination of SP with IP demonstrated a substantial increase in electroosmotic flow. The synergistic effects of SP were attributed to the negative change in net charge of the skin in addition to the increased size and number of permeation pathways. Despite these encouraging results, the authors highlighted that minimal prior research examined the effects of SP and IP on electro-osmosis and that their study alone was insufficient to circumstantiate the observed synergistic effects.

\section{Ultrasound-based transdermal delivery using nanoparticles}

Recently, the use of nanoparticles has revolutionized how drugs are formulated and delivered. Some of the developed nanoformulations that have been investigated for transdermal use include polymeric nanoparticles, ${ }^{47}$ nanogels, ${ }^{48}$ nanoemulsions,${ }^{49}$ lipid-based nanoparticles,${ }^{50}$ dendrimers, ${ }^{51}$ nanocapsules, ${ }^{52}$ and nanosponges. ${ }^{53}$ Each of these 
nanoparticles hold varying specialized properties due to enhanced pharmacokinetic attributes, improved bioavailability, and improved drug targeting. ${ }^{53}$ The administration of nanotherapeutics is a focal area of interest, with successful implementation in the treatment of diseases such as cancer, hepatitis, and neurological and autoimmune diseases. ${ }^{53}$ The incorporation of nanomedicine into sonophoretic delivery is one branch of current research, with lipid-based nanocarriers being one of the potential nanoformulations. A recent study by Rangsimawong et $\mathrm{al}^{54}$ examined the effects of lowfrequency SP $(20 \mathrm{kHz})$ for the percutaneous transport of sodium fluorescein into porcine skin in vitro using liposomes, niosomes (nonionic surfactant-based vesicle), and solid lipid nanoparticles (carrier consisting of a solid lipid core matrix). The skin samples were then inspected using confocal laser scanning microscopy, Fourier transform infrared spectroscopy, and scanning electron microscopy. The collected results indicated that the sonophoretic delivery of niosomes and solid lipid particles did not improve the flux rate of sodium fluorescein into the skin, with the insonified solid lipid nanoparticle sample being significantly lower than the sample without SP. Only the sonophoretic liposome containing sample displayed an improvement in flux rate, but this increase was not significant. These results were likely attributed to the effects of sonication breaking the vesicles, lamellae, and solid cores of the three nanocarriers before they could reach the skin surface. However, an intriguing coincidental finding relating to liposomes was identified. As the liposome lamellae was broken down, the remaining phospholipids diffused into the SC to coactively repair the skin through adsorption (Figure 10) and also enhanced drug penetration.

Manikkath et $\mathrm{al}^{55}$ investigated an alternative potential for the sonophoretic delivery of drugs through nanoparticles by testing the permeability of ketoprofen with peptide dendrimers on mouse skin in vitro and in vivo and comparing these results with that of orally administered ketoprofen. Peptide dendrimers are hyperbranched nanopolymeric structures with a variety of possible charges. ${ }^{53}$ The examined dendrimer charges were $4^{+}, 8^{+}$, and $16^{+}$. The in vitro and in vivo studies displayed similar results, both indicating that the insonified $16^{+}$dendrimer complex sample induced greater permeation of ketoprofen. The pretreatment of the $16^{+}$dendrimer complex, followed by SP in the in vitro study, had a remarkable enhancement ratio of 1,369.15 over the control of passive diffusion. This transdermal permeation was also significantly higher than that of SP alone (Figure 11A). Ketoprofen permeation in the simultaneous application of
SP and peptide dendrimers was found to be less than the pretreated sample (Figure 11B). Furthermore, the in vivo insonified peptide dendrimer samples displayed no dermal toxicity. They concluded that the sonophoretic application of pretreated peptide dendrimers possesses great potential as a route for transdermal drug delivery as it is safe and holds a comparable potential drug plasma level to that of the oral medication of ketoprofen.

While some nanoparticles may hold potential efficacy for ultrasound-based drug transport, the previously mentioned nanomaterials were not acoustically active. As a consequence, there remained an inherent limitation to the effectiveness of sonophoretic application. It is for this reason that some of the investigated nanoparticles were not deemed competent to efficiently transport drugs through SP. Cup-shaped polymeric nanoparticles have also been used in ultrasound-mediated transdermal delivery. ${ }^{56}$ The nanocup consists of a single-cavity polymeric nanoparticle, which is ultrasound responsive and traps a bubble in its cavity. Ultrasound exposure to the nanocups generates cavitation activity, which causes active micropumping of the suspended components. These detached microbubbles consequently collapse due to cavitation and release the therapeutic agents into the surrounding tissue. To determine the potential of this nanoparticle in ultrasound, Bhatnagar et $\mathrm{al}^{56}$ investigated the sonophoretic application of nanocups containing ovalbumin into porcine skin in vivo and ex vivo. By using a frequency of $256 \mathrm{kHz}$ in a pulsed mode with a duty cycle of $10 \%$, their ex vivo study demonstrated that the use of a hydrogel loaded with nanocups containing ovalbumin allowed for a marked increase in penetration depth as shown in Figure 12A. The application of nanocup-loaded gel without ultrasound and treatment of ovalbumin gel with ultrasound resulted in penetration depths of $100 \mu \mathrm{m}$, while the pretreatment of hydrogel containing nanocups with subsequent administration of ovalbumin led to the majority of ovalbumin passively diffusing into the superficial layers of the skin. Simultaneous use of nanocup-loaded hydrogel with ultrasound induced a significant increase in penetration depth of ovalbumin to $550 \mu \mathrm{m}$ through active transport by nanocup micropumping. The authors established that the simultaneous use of loaded nanocups with ultrasound displayed significant increases in volume of ovalbumin deposited into tissue when compared to the application of the chemical penetration enhancer sodium dodecyl sulphate, in addition to the previously utilized configurations (Figure 12B). By collecting blood samples of the treated mice, they were also able to determine that the 

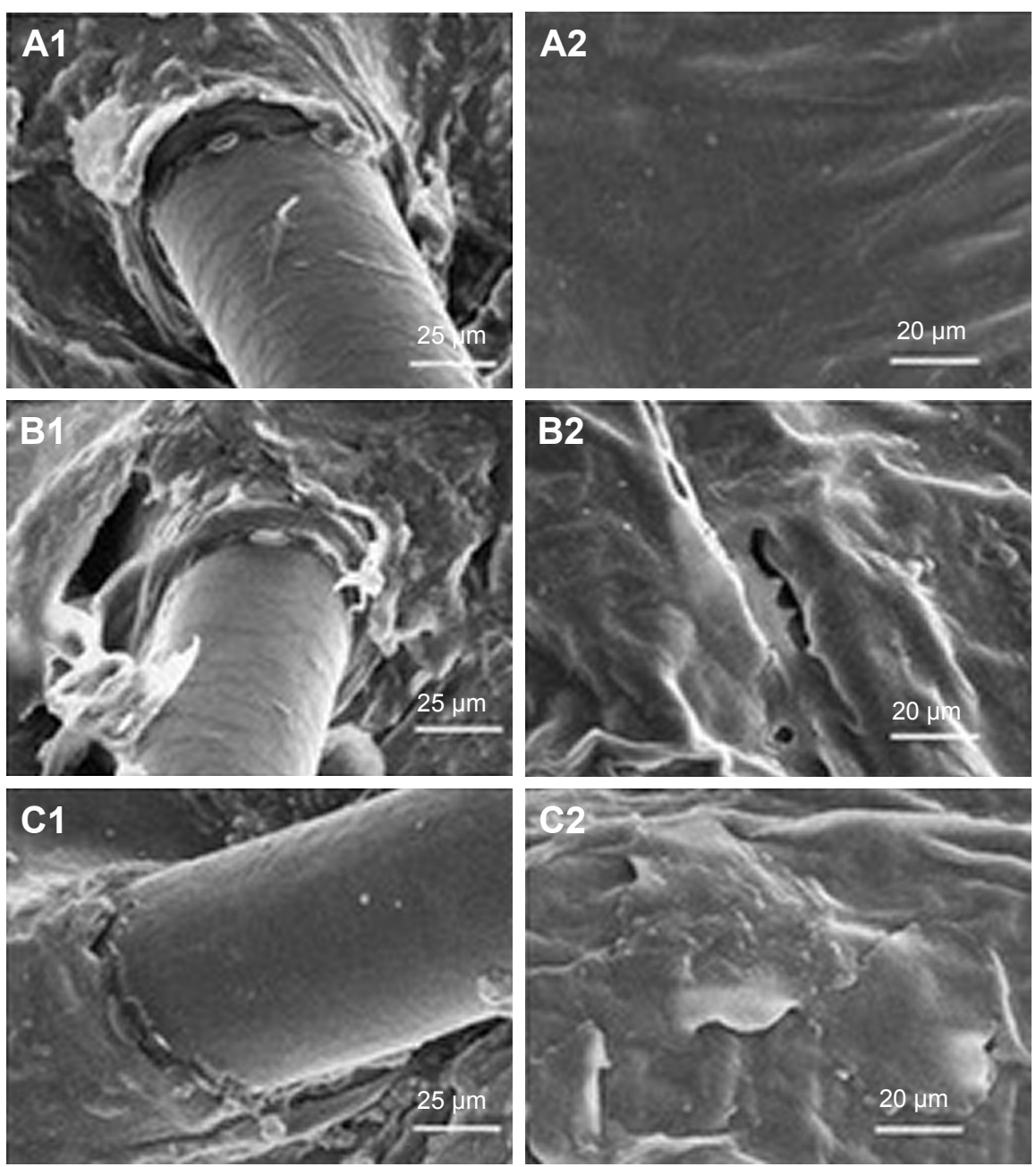

Figure 10 Scanning electron microscopy images of porcine skin.

Notes: (A) Control sample without sonophoresis, (B) sonophoretic sample $(20 \mathrm{kHz})$, and $(\mathbf{C})$ sonophoretic sample with liposomes (20 kHz). Reproduced with permission from Taylor and Francis (http://www.tandfonline.com). Rangsimawong W, Opanasopit P, Rojanarata T, Panomsuk S, Ngawhirunpat T. Influence of sonophoresis on transdermal drug delivery of hydrophilic compound-loaded lipid nanocarriers. Pharmaceutical Development and Technology. 2017;22(4):597-605. ${ }^{54}$

A

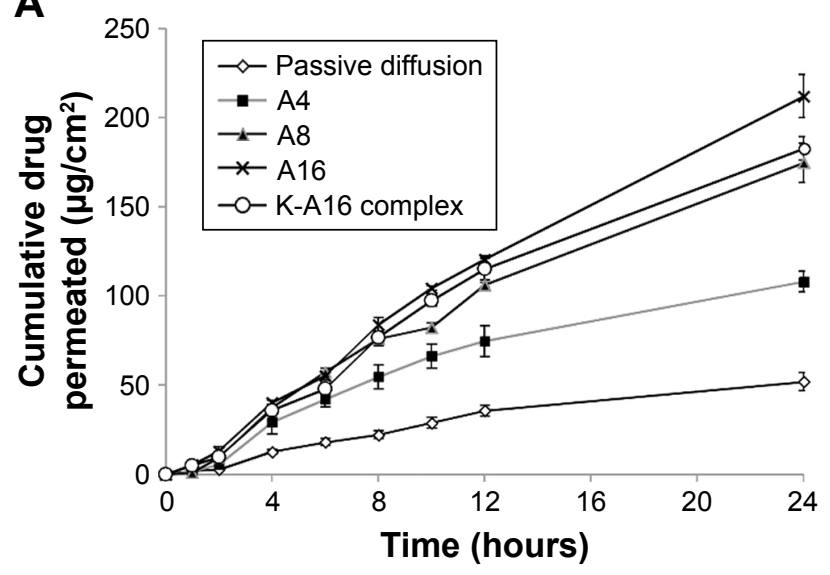

B

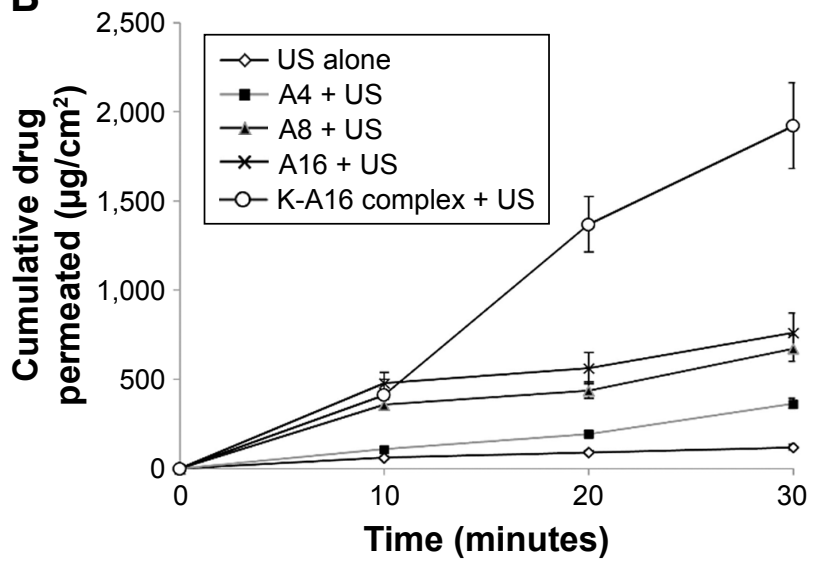

Figure I I (A) In vitro permeation profiles of ketoprofen with pretreated peptide dendrimers followed by sonophoresis. (B) In vitro permeation profiles of ketoprofen with the simultaneous application of peptide dendrimers and sonophoresis.

Note: Reproduced from International Journal of Pharmaceutics, 52I(I-2), Manikkath J, Hegde AR, Kalthur G, Parekh HS, Mutalik S, Influence of peptide dendrimers and sonophoresis on the transdermal delivery of ketoprofen. International journal of pharmaceutics, I10-119. Copyright (2017), with permission from Elsevier. ${ }^{38}$

Abbreviation: US, ultrasound. 

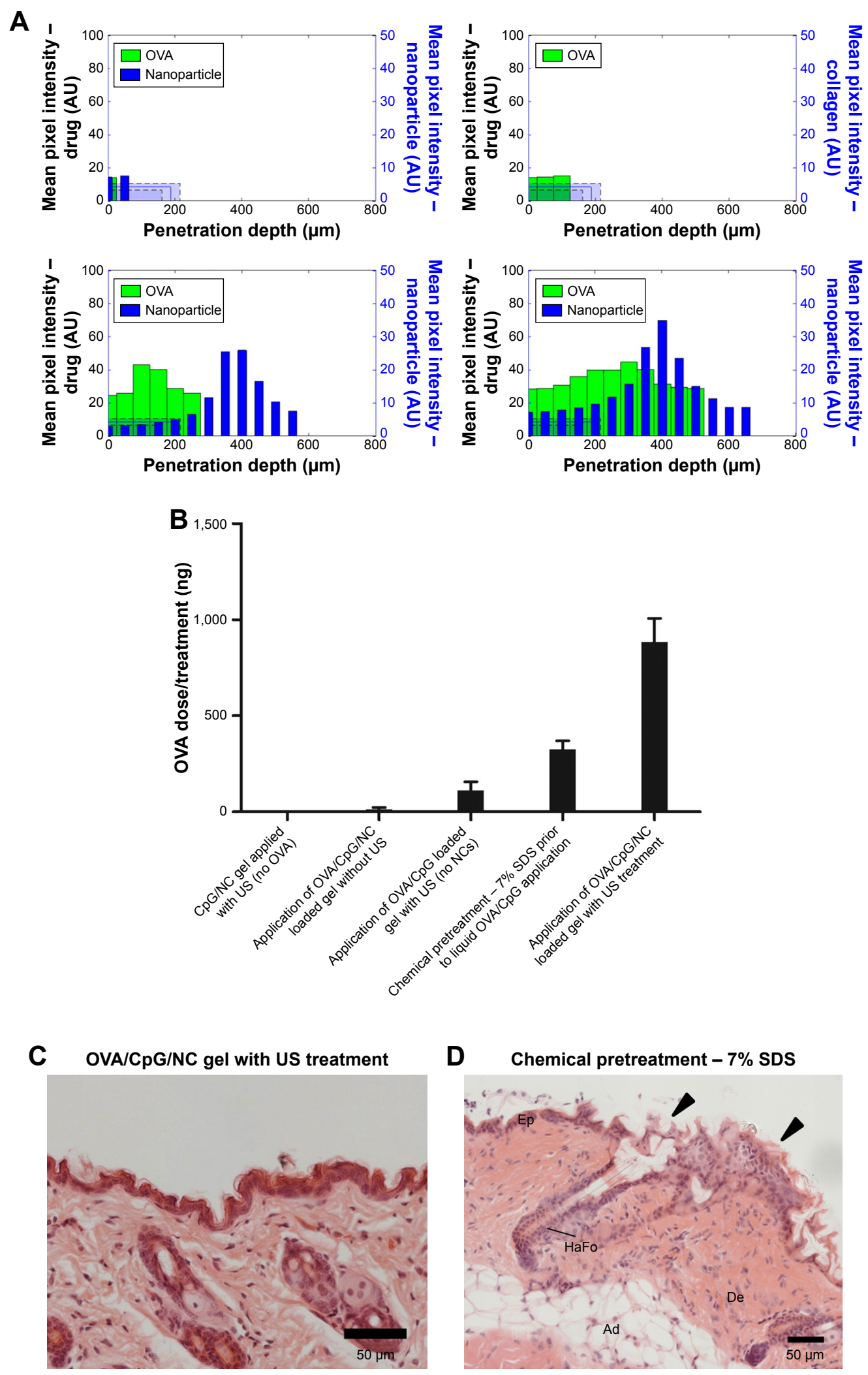

D Chemical pretreatment $-7 \%$ SDS

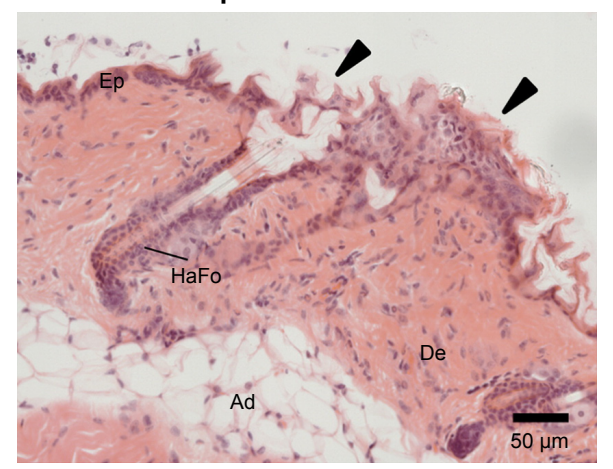

Figure 12 (A) Distribution profiles of nanocups and ovalbumin, with mean pixel intensity against penetration depth ( $\mu \mathrm{m})$ for various treatment configurations. (B) Dose (ng) of ovalbumin transdermally transported for various treatment configurations. (C) Optical density of anti-ovalbumin lgG antibody levels for various treatment configurations. (D) Histological images of the nanocup sonophoresis group and the chemical pretreatment group.

Note: Reproduced from Bhatnagar S, Kwan JJ, Shah AR, Coussios CC, Carlisle RC. Exploitation of sub-micron cavitation nuclei to enhance ultrasound-mediated transdermal transport and penetration of vaccines. J Control Release. 2016;238:22-30. ${ }^{56}$

Abbreviations: NC, nanocup; OVA, ovalbumin; US, ultrasound; Ep, epidermis; De, dermis; Ad, adipose tissue; HaFo, hair follicle. 
simultaneous sonophoretic treatment with loaded nanocups were able to induce a significant immune response greater than the chemical penetration enhancer group, although not as effective as the subcutaneous injection control group (Figure 12C). In subsequent histological examinations of excised murine skin, they found that nanocup SP did not result in structural damage to the $\mathrm{SC}$, unlike the chemical penetration enhancer group (Figure 12D).

\section{Challenges and conclusion}

While the discussed studies have examined the capability and safety of ultrasound-mediated transdermal drug delivery, there are still several limitations that need to be addressed regarding the influence of modifiable parameters such as frequency, duty cycle, the coupling medium, and pressure amplitude. Much of the current research utilizes and records a diverse assortment of ultrasound parameters and epidermal targets. Furthermore, not all studies quantitatively specify other important determining factors such as transducer size, the type and amount of coupling medium used, and the processing of examined epidermal samples. ${ }^{27}$ Further quantitative research into understanding these parameters is required to thoroughly optimize the process of transdermal drug delivery and prevent irreversible damage. In addition, there is insufficient research regarding the prolonged use of low-frequency SP. Much of the current research examines the acute effects of SP and predominantly uses in vitro samples, ${ }^{57}$ but many patients suffer from chronic conditions and may be required to obtain various drugs daily. Additional in vivo studies examining repeated use of low-frequency SP are essential to ensure the safety of all patients. When combining the potential variable influence of the transported compound through distinctive molecular sizes and structures, the comparison of results can become somewhat limited, especially when the underlying mechanisms of acoustic cavitation in vivo are still not precisely understood.

It is also important to consider the bioeffects and associated safety issues of ultrasound-mediated transdermal delivery. Ultrasound frequency profoundly affects the integral process of acoustic cavitation, but the consequential bioeffects of SP are also predominantly dependent on the appointed frequency of operation. Observed thermal effects of increasing frequency on tissue in vivo precipitate a proportional increase in generated temperature. ${ }^{58}$ Disregard for the gradual accumulation of heat, particularly in situations with a calculated high thermal index, may cause harmful effects on skin, such as superficial burns, epidermal detachment, and the coagulative necrosis of tissue in extreme cases..$^{58,59}$ The suggested temperature threshold for ultrasound to avoid thermal damage is $43^{\circ} \mathrm{C}$, with a maximum temperature increase of $10^{\circ} \mathrm{C} .^{12}$

For the practical application of SP, controlled cavitation is necessary to avoid unnecessary excess and possibly permanent damage to the skin or underlying tissue through hemorrhage, cellular death, or thrombosis. ${ }^{60}$ A study conducted by Boucaud et $\mathrm{al}^{61}$ demonstrated that although there was no superficial damage using low-frequency ultrasound with a cavitation medium on rats in vivo, histologically observable delayed bioeffects of lesions and necrosis in muscle tissue were discovered 24 hours after ultrasound exposure. The established threshold for provoking lesions on human skin in vitro from cavitation-related events is delineated to be $2.5 \mathrm{Wcm}^{-2}$ at $20 \mathrm{kHz}$, with a 1-hour exposure for pulsed ultrasound and 10 minutes for continuous wave ultrasound..$^{58}$ Inertial cavitation may also consequently produce reactive free radicals from thermolysis. ${ }^{60}$ While it is speculated that recombination of free radicals may cause genetic mutations, it has been difficult to induce this DNA strand damage through ultrasound in vitro.

Within the nanoparticle domain of drug delivery, there are various inherent pharmacological issues and safety challenges that have been identified. Due to their size, nanoparticles readily diffuse through biological barriers within the body and therefore cannot be treated as traditional macromolecular drugs. As previously discussed, there is an incredibly broad range of particles within nanotherapeutics. Small compositional changes to a nanoparticle, such as particle structure and surface properties, alter its biodistribution and clearance in vivo. ${ }^{62}$ In addition, each nanoparticle has its own potential for nanoparticle toxicity that must be thoroughly investigated. Particles that cannot be excreted or metabolized efficiently may accumulate and induce adverse effects. ${ }^{63}$

Despite the current and future research into this drug delivery technique, a continuous challenge is the further development of a sonophoretic device. The prominent difficulties in developing an improved sonophoretic device lie in improving device portability and reducing device cost, while maintaining or improving sonophoretic potential. ${ }^{64}$ Most of the transdermal studies currently utilize the device marketed by "Echo Therapeutics" called "SonoPrep". While this device has been approved for transdermal use by the US Food and Drug Administration, ${ }^{57}$ it is not commercially available. Furthermore, efficient incorporation of dual-frequency SP or additional transdermal drug delivery techniques to 
maximize percutaneous transport further exacerbates current complications and challenges within device development. Sonophoretic devices are being utilized within particular innovative clinics for skin care, but it has yet to be implemented into the greater health care system. The future of ultrasound-mediated transdermal delivery is promising. With the growing understanding of SP for percutaneous delivery and the continued efforts of medical doctors, engineers, and scientists to advance this technology, it is likely that ultrasound-based transdermal delivery platform will be a step closer to clinical applications.

\section{Disclosure}

The authors report no conflicts of interest in this work.

\section{References}

1. Alkilani AZ, Mccrudden MT, Donnelly RF. Transdermal Drug Delivery: Innovative Pharmaceutical Developments Based on Disruption of the Barrier Properties of the stratum corneum. Pharmaceutics. 2015;7(4):438-470.

2. Schoellhammer CM, Blankschtein D, Langer R. Skin permeabilization for transdermal drug delivery: recent advances and future prospects. Expert Opin Drug Deliv. 2014;11(3):393-407.

3. Liao AH, Ho HC, Lin YC, Chen HK, Wang CH. Effects of microbubble size on ultrasound-Induced Transdermal Delivery of High-MolecularWeight Drugs. PLoS One. 2015;10(9):e0138500.

4. Prausnitz MR, Langer R. Transdermal drug delivery. Nat Biotechnol. 2008;26(11):1261-1268.

5. Solé R, Conde-Pueyo N. Ultrasound approach tracks gut microbes. Nature. 2018;553(7686):36-37.

6. Abu-Zidan FM, Hefny AF, Corr P. Clinical ultrasound physics. JEmerg Trauma Shock. 2011;4(4):501.

7. Sites BD, Brull R, Chan VW, et al. Artifacts and pitfall errors associated with ultrasound-guided regional anesthesia. Part I: understanding the basic principles of ultrasound physics and machine operations. Reg Anesth Pain Med. 2007;32(5):412-418.

8. Jain A, Swaminathan M. Physics of Ultrasound. Anaesthesia Pain Intensive Care. 2015;19(4):533-539.

9. Shipp T. Basic Principles and Safety of Diagnostic Ultrasound in Obstetrics and Gynecology. Alphen aan den Rijn: Wolters Kluwer; 2017.

10. Martin DJ, Wells ITP, Goodwin CR. Physics of ultrasound. Anaesthesia Intensive Care Medicine. 2015;16(3):132-135.

11. Haar G. Ultrasonic imaging: safety considerations. Interface Focus. 2011;1(4):686.

12. Haar G. Ultrasound bioeffects and safety. Proceedings of the Institution of Mechanical Engineers, Part H. J Eng Med. 2010;224(2):363-373.

13. Stride EP, Coussios CC. Cavitation and contrast: the use of bubbles in ultrasound imaging and therapy. Proc Inst Mech Eng H. 2010; 224(2):171-191.

14. Ashokkumar M. The characterization of acoustic cavitation bubbles - an overview. Ultrason Sonochem. 2011;18(4):864-872.

15. de Cock I, Zagato E, Braeckmans K, et al. Ultrasound and microbubble mediated drug delivery: acoustic pressure as determinant for uptake via membrane pores or endocytosis. J Control Release. 2015;197:20-28.

16. Wolloch L, Kost J. The importance of microjet vs shock wave formation in sonophoresis. J Control Release. 2010;148(2):204-211.

17. Chan Kim Y, Min H, Yu J, et al. Forced infiltration of silica beads into densely-packed glass fibre beds for thin composite laminates. RSC Adv. 2016;6(94):91341-91348.

18. Ita K. Recent progress in transdermal sonophoresis. Pharm Dev Technol. 2017;22(4):458-466.
19. Holland CK, Apfel RE. An improved theory for the prediction of microcavitation thresholds. IEEE Trans Ultrason Ferroelectr Freq Control. 1989;36(2):204-208.

20. Kollmann C, ter Haar G, Dolezal L, Hennerici M, Salvesen KA, Valentin L. Ultrasound Output: thermal (TI) and mechanical (MI) indices. Ultraschall in der Medizin. 2013:34(5);422-434.

21. Şen T, Tüfekçioğlu O, Koza Y. Mechanical index. Anatol J Cardiol. 2015;15(4):334-336.

22. Wing M. Phonophoresis with hydrocortisone in the treatment of temporomandibular joint dysfunction. Phys Ther. 1982;62(1):32-33.

23. Azagury A, Khoury L, Enden G, Kost J. Ultrasound mediated transdermal drug delivery. Adv Drug Deliv Rev. 2014;72:127-143.

24. Park D, Park H, Seo J, Lee S. Sonophoresis in transdermal drug deliverys. Ultrasonics. 2014;54(1):56-65.

25. Canavese G, Ancona A, Racca L, et al. Nanoparticle-assisted ultrasound: A special focus on sonodynamic therapy against cancer. Chem Eng J. 2018;340:155-172.

26. Park D, Song G, Jo Y, et al. Sonophoresis Using Ultrasound Contrast Agents: Dependence on Concentration. PLoS One. 2016;11(6): e0157707.

27. Rao R, Nanda S. Sonophoresis: recent advancements and future trends. J Pharm Pharmacol. 2009;61(6):689-705.

28. Feiszthuber H, Bhatnagar S, Gyöngy M, Coussios CC. Cavitationenhanced delivery of insulin in agar and porcine models of human skin. Phys Med Biol. 2015;60(6):2421-2434.

29. Jabbari N, Asghari MH, Ahmadian H, Mikaili P. Developing a Commercial Air Ultrasonic Ceramic Transducer to Transdermal Insulin Delivery. J Med Signals Sens. 2015;5(2):117-122.

30. Yu ZW, Liang Y, Liang WQ. Low-frequency sonophoresis enhances rivastigmine permeation in vitro and in vivo. Pharmazie. 2015;70(6): 379-380.

31. Pereira TA, Ramos DN, Lopez RF. Hydrogel increases localized transport regions and skin permeability during low frequency ultrasound treatment. Sci Rep. 2017;7:44236.

32. Aldwaikat M, Alarjah M. Investigating the sonophoresis effect on the permeation of diclofenac sodium using 3D skin equivalent. Ultrason Sonochem. 2015;22:580-587.

33. Schoellhammer CM, Polat BE, Mendenhall J, et al. Rapid skin permeabilization by the simultaneous application of dual-frequency, high-intensity ultrasound. J Control Release. 2012;163(2):154-160.

34. Schoellhammer CM, Srinivasan S, Barman R, et al. Applicability and safety of dual-frequency ultrasonic treatment for the transdermal delivery of drugs. J Control Release. 2015;202:93-100.

35. Yin L, Qin FH, Zhou Y, Qi X. Enhancing percutaneous permeability of sinomenine hydrochloride using dual-frequency sonophoresis. J Drug Deliv Sci Technol. 2016;36:62-67.

36. Zhang Y, Zhang Y, Li S. Combination and simultaneous resonances of gas bubbles oscillating in liquids under dual-frequency acoustic excitation. Ultrason Sonochem. 2017;35(Pt A):431-439.

37. Zhang Y, Li S. Acoustical scattering cross section of gas bubbles under dual-frequency acoustic excitation. Ultrason Sonochem. 2015; 26:437-444.

38. Manikkath J, Hegde AR, Kalthur G, Parekh HS, Mutalik S. Influence of peptide dendrimers and sonophoresis on the transdermal delivery of ketoprofen. International Journal of Pharmaceutics. 2017 Apr 15; 521(1-2):110-119.

39. Al-Qallaf B, Das Diganta B. Optimizing microneedle arrays to increase skin permeability for transdermal drug delivery. Ann N Y Acad Sci. 2009; 1161(1):83-94.

40. Haj-Ahmad R, Khan H, Arshad M, et al. Microneedle Coating Techniques for Transdermal Drug Delivery. In. Vol 7. Basel: MDPI AG; 2015:486-502.

41. Zorec B, Becker S, Reberšek M, Miklavčič D, Pavšelj N. Skin electroporation for transdermal drug delivery: the influence of the order of different square wave electric pulses. Int J Pharm. 2013;457(1):214-223.

42. Roustit M, Blaise S, Cracowski JL. Trials and tribulations of skin iontophoresis in therapeutics. Br J Clin Pharmacol. 2014;77(1):63-71. 
43. Ita K. Transdermal iontophoretic drug delivery: advances and challenges. J Drug Target. 2016;24(5):386-391.

44. Pamornpathomkul B, Duangjit S, Laohapatarapant S, Rojanarata T, Opanasopit P, Ngawhirunpat T. Transdermal delivery of fluorescein isothiocyanate-dextrans using the combination of microneedles and lowfrequency sonophoresis. Asian J Pharm Sci. 2015;10(5):415-424.

45. Han T, Das DB. Potential of combined ultrasound and microneedles for enhanced transdermal drug permeation: a review. Eur J Pharm Biopharm. 2015;89:312-328.

46. Tokumoto S, Higo N, Todo H, Sugibayashi K. Effect of combination of low-frequency sonophoresis or electroporation with iontophoresis on the mannitol flux or electroosmosis through excised skin. Biol Pharm Bull. 2016;39(7):1206-1210.

47. Kang E, Min HS, Lee J, et al. Nanobubbles from gas-generating polymeric nanoparticles: ultrasound imaging of living subjects. Angew Chem Int Ed Engl. 2010;49(3):524-528.

48. Hamidi M, Azadi A, Rafiei P. Hydrogel nanoparticles in drug delivery. Adv Drug Deliv Rev. 2008;60(15):1638-1649.

49. Shakeel F, Ramadan W. Transdermal delivery of anticancer drug caffeine from water-in-oil nanoemulsions. Colloids Surf B Biointerfaces. 2010;75(1):356-362.

50. Dahlan A, Alpar HO, Murdan S. An investigation into the combination of low frequency ultrasound and liposomes on skin permeability. Int J Pharm. 2009;379(1):139-142.

51. Huang B, Dong WJ, Yang GY, Wang W, Ji CH, Zhou FN. Dendrimercoupled sonophoresis-mediated transdermal drug-delivery system for diclofenac. Drug Des Devel Ther. 2015;9:3867-3876.

52. Varshosaz J, Hajhashemi V, Soltanzadeh S. Lipid nanocapsule-based gels for enhancement of transdermal delivery of ketorolac tromethamine. J Drug Deliv. 2011;2011:1-7.

53. Prasad M, Lambe UP, Brar B, et al. Nanotherapeutics: An insight into healthcare and multi-dimensional applications in medical sector of the modern world. Biomed Pharmacother. 2018;97:1521-1537.

54. Rangsimawong W, Opanasopit P, Rojanarata T, Panomsuk S, Ngawhirunpat T. Influence of sonophoresis on transdermal drug delivery of hydrophilic compound-loaded lipid nanocarriers. Pharm Dev Technol. 2017;22(4):597-605.
55. Manikkath J, Hegde AR, Kalthur G, Parekh HS, Mutalik S. Influence of peptide dendrimers and sonophoresis on the transdermal delivery of ketoprofen. Int J Pharm. 2017;521(1-2):110-119.

56. Bhatnagar S, Kwan JJ, Shah AR, Coussios CC, Carlisle RC. Exploitation of sub-micron cavitation nuclei to enhance ultrasound-mediated transdermal transport and penetration of vaccines. $J$ Control Release. 2016;238:22-30.

57. Polat BE, Blankschtein D, Langer R. Low-frequency sonophoresis: application to the transdermal delivery of macromolecules and hydrophilic drugs. Expert Opin Drug Deliv. 2010;7(12):1415-1432.

58. Ahmadi F, Mcloughlin IV, Chauhan S, Ter-Haar G. Bio-effects and safety of low-intensity, low-frequency ultrasonic exposure. Prog Biophys Mol Biol. 2012;108(3):119-138.

59. Miller DL, Smith NB, Bailey MR, et al. Overview of therapeutic ultrasound applications and safety considerations. J Ultrasound Med.2012 31(4):623-634.

60. Qin P, Han T, Yu ACH, Xu L. Mechanistic understanding the bioeffects of ultrasound-driven microbubbles to enhance macromolecule delivery. J Control Release. 2018;272:169-181.

61. Boucaud A, Montharu J, Machet L, et al. Clinical, histologic, and electron microscopy study of skin exposed to low-frequency ultrasound. Anat Rec. 2001;264(1):114-119.

62. Desai N. Challenges in development of nanoparticle-based therapeutics. AAPS J. 2012;14(2):282-295.

63. Zolnik BS, González-Fernández A, Sadrieh N, Dobrovolskaia MA. Nanoparticles and the immune system. Endocrinology. 2010;151(2): 458-465.

64. Boucaud A. Trends in the use of ultrasound-mediated transdermal drug delivery. Drug Discov Today. 2004;9(19):827-828.

65. Petchsangsai M, Rojanarata T, Opanasopit P, Ngawhirunpat T. The Combination of Microneedles with Electroporation and Sonophoresis to Enhance Hydrophilic Macromolecule Skin Penetration. Biol Pharm Bull. 2014;37(8):1373-1382.

66. Van Smeden J, Janssen M, Goori GS, Bouwstra JA. The important role of stratum corneum lipids for the cutaneous barrier function. Biochimica et Biophysica Acta (BBA)-Molecular and Cell Biology of Lipids. 2014;1841(3):295-313.
International Journal of Nanomedicine

\section{Publish your work in this journal}

The International Journal of Nanomedicine is an international, peerreviewed journal focusing on the application of nanotechnology in diagnostics, therapeutics, and drug delivery systems throughout the biomedical field. This journal is indexed on PubMed Central, MedLine, CAS, SciSearch ${ }^{\circledR}$, Current Contents ${ }^{\circledR} /$ Clinical Medicine,

\section{Dovepress}

Journal Citation Reports/Science Edition, EMBase, Scopus and the Elsevier Bibliographic databases. The manuscript management system is completely online and includes a very quick and fair peer-review system, which is all easy to use. Visit http://www.dovepress.com/ testimonials.php to read real quotes from published authors. 